\title{
Comment on "on the stability of quadratic double centralizers and quadratic multipliers: a fixed point approach" [Bodaghi et al., j. inequal. appl. 2011, article id 957541 (2011)]
}

Choonkil Park', Jung Rye lee ${ }^{2}$, Dong Yun Shin ${ }^{3}$ and Madjid Eshaghi Gordji $4^{*}$

* Correspondence: madjid. eshaghi@gmail.com

${ }^{4}$ Department of Mathematics, Semnan University, P. O. Box 35195-363, Semnan, Iran Full list of author information is available at the end of the article

\begin{abstract}
Bodaghi et al. [On the stability of quadratic double centralizers and quadratic multipliers: a fixed point approach. J. Inequal. Appl. 2011, Article ID 957541, 9pp. (2011)] proved the Hyers-Ulam stability of quadratic double centralizers and quadratic multipliers on Banach algebras by fixed point method. One can easily show that all the quadratic double centralizers $(L, R)$ in the main results must be $(0,0)$. The results are trivial. In this article, we correct the results.

2010 MSC: 39B52; 46H25; 47H10; 39B72.
\end{abstract}

Keywords: quadratic functional equation, multiplier, double centralizer, stability, superstability

\section{Introduction}

In 1940, Ulam [1] raised the following question concerning stability of group homomorphisms: Under what condition does there exist an additive mapping near an approximately additive mapping? Hyers [2] answered the problem of Ulam for Banach spaces. He showed that for Banach spaces $\mathcal{X}$ and $\mathcal{Y}$, if $\varepsilon>0$ and $f: \mathcal{X} \rightarrow \mathcal{Y}$ such that

$$
\|f(x+y)-f(x)-f(y)\| \leq \varepsilon
$$

for all $x, y \in \mathcal{X}$, then there exists a unique additive mapping $T: \mathcal{X} \rightarrow \mathcal{Y}$ such that

$$
\|f(x)-T(x)\| \leq \varepsilon \quad(x \in \mathcal{X}) .
$$

Consider $f: \mathcal{X} \rightarrow \mathcal{Y}$ to be a mapping such that $f(t x)$ is continuous in $t \in \mathbb{R}$ for all $x \in \mathcal{X}$. Assume that there exist constant $\varepsilon \geq 0$ and $p \in[0,1)$ such that

$$
\|f(x+y)-f(x)-f(y)\| \leq \varepsilon\left(\left\|x||^{p}+\right\| y||^{p}\right) \quad(x \in \mathcal{X})
$$

Rassias [3] showed that there exists a unique $\mathbb{R}$-linear mapping $T: \mathcal{X} \rightarrow \mathcal{Y}$ such that

$$
\|f(x)-T(x)\| \leq \frac{2 \varepsilon}{2-2^{p}} \| x||^{p} \quad(x \in \mathcal{X}) .
$$

(C) 2011 Park et al; licensee Springer. This is an Open Access article distributed under the terms of the Creative Commons Attribution License (http://creativecommons.org/licenses/by/2.0), which permits unrestricted use, distribution, and reproduction in any medium, provided the original work is properly cited. 
Găvruta [4] generalized the Rassias' result. A square norm on an inner product space satisfies the important parallelogram equality

$$
\left\|x+y||^{2}+\right\| x-y||^{2}=2\left(\left\|x||^{2}+\right\| y||^{2}\right) .
$$

Recall that the functional equation

$$
f(x+y)+f(x-y)=2 f(x)+2 f(y)
$$

is called a quadratic functional equation. In particular, every solution of the functional equation (1.1) is said to be a quadratic mapping. A Hyers-Ulam stability problem for the quadratic functional equation was proved by Skof [5] for mappings $f: \mathcal{X} \rightarrow \mathcal{Y}$, where $\mathcal{X}$ is a normed space and $\mathcal{Y}$ is a Banach space. Cholewa [6] noticed that the theorem of Skof is still true if the relevant domain $E_{1}$ is replaced by an Abelian group. Indeed, Czerwik [7] proved the Hyers-Ulam stability of the quadratic functional equation. Since then, the stability problems of various functional equation have been extensively investigated by a number of authors [8-20].

\section{Stability of quadratic double centralizers}

A linear mapping $L: \mathcal{A} \rightarrow \mathcal{A}$ is said to be left centralizer on $\mathcal{A}$ if $L(a b)=L(a) b$ for all $a, b \in \mathcal{A}$. Similarly, a linear mapping $R: \mathcal{A} \rightarrow \mathcal{A}$ satisfying that $R(a b)=a R(b)$ for all $a, b \in \mathcal{A}$ is called right centralizer on $\mathcal{A}$. A double centralizer on $\mathcal{A}$ is a pair $(L, R)$, where $L$ is a left centralizer, $R$ is a right centralizer and $a L(b)=R(a) b$ for all $a, b \in \mathcal{A}$. An operator $T: \mathcal{A} \rightarrow \mathcal{A}$ is said to be a multiplier if $a T(b)=T(a) b$ for all $a, b \in \mathcal{A}$.

Throughout this article, let $\mathcal{A}$ be a complex Banach algebra. Recall that a mapping $L: \mathcal{A} \rightarrow \mathcal{A}$ is a quadratic left centralizer if $L$ is a quadratic homogeneous mapping, that is quadratic and $L(\lambda a)=\lambda^{2} L(a)$ for all $a \in \mathcal{A}$ and $\lambda \in \mathbb{C}$ and $L(a b)=L(a) b^{2}$ for all $a, b \in \mathcal{A}$, and a mapping $R: \mathcal{A} \rightarrow \mathcal{A}$ is a quadratic right centralizer if $R$ is a quadratic homogeneous mapping and $R(a b)=a^{2} R(b)$ for all $a, b \in \mathcal{A}$. Also a quadratic double centralizer of an algebra $\mathcal{A}$ is a pair $(L, R)$, where $L$ is a quadratic left centralizer, $R$ is a quadratic right centralizer and $a^{2} L(b)=R(a) b^{2}$ for all $a, b \in \mathcal{A}$ (see [21] for details).

It is proven in [8] that for vector spaces $\mathcal{X}$ and $\mathcal{Y}$ and a fixed positive integer $k$, a mapping $f: \mathcal{X} \rightarrow \mathcal{Y}$ is quadratic if and only if the following equality holds:

$$
2 f\left(\frac{k x+k y}{2}\right)+2 f\left(\frac{k x-k y}{2}\right)=k^{2} f(x)+k^{2} f(y)
$$

We thus can show that $f$ is quadratic if and only if for a fixed positive integer $k$, the following equality holds:

$$
f(k x+k y)+f(k x-k y)=2 k^{2} f(x)+2 k^{2} f(y) .
$$

Before proceeding to the main results, we will state the following theorem which is useful to our purpose.

Theorem 2.1. (The alternative of fixed point [22]). Suppose that we are given a complete generalized metric space $(X, d)$ and a strictly contractive mapping $T: X \rightarrow X$ with Lipschitz constant L. Then, for each given $x \in X$, either $d\left(T^{n} x, T^{n+1} x\right)=\infty$ for all $n \geq 0$ or other exists a natural number $n_{0}$ such that 
(i) $d\left(T^{n} x, T^{n+1} x\right)<\infty$ for all $n \geq n_{0}$;

(ii) the sequence $\left\{T^{n} x\right\}$ is convergent to a fixed point $y^{*}$ of $T$;

(iii) $y^{*}$ is the unique fixed point of $T$ in the set $\Lambda=\left\{y \in X: d\left(T^{n_{0}} x, y\right)<\infty\right\}$;

(iv) $d\left(y, y^{*}\right) \leq \frac{1}{1-L} d(y, T y)$ for all $y \in \Lambda$.

Theorem 2.2. Let $f_{j}: \mathcal{A} \rightarrow \mathcal{A}$ be continuous mappings with $f_{j}(0)=0(j=0,1)$, and let $\phi: \mathcal{A}^{4} \rightarrow[0, \infty)$ be continuous in the first and second variables such that

$$
\begin{aligned}
& \left\|f_{j}(\lambda a+\lambda b)+f_{j}(\lambda a-\lambda b)-2 \lambda^{2}\left[f_{j}(a)+f_{j}(b)\right]\right\| \leq \phi(a, b, 0,0) \\
& \begin{aligned}
\| f_{j}(c d) & -\left[(1-j)\left(f_{j}(c) d^{2}\right)^{1-j}+j\left(c^{2} f_{j}(d)\right)^{j}\right]+u^{2} f_{0}(v)-f_{1}(u) v^{2} \| \\
& \leq \phi(c, d, u, v)
\end{aligned}
\end{aligned}
$$

for all $\lambda \in \mathbb{T}=\{\lambda \in \mathbb{C}:|\lambda|=1\}$ and all $a, b, c, d, u, v \in \mathcal{A}, j=0$, 1. If there exists a constant $m, 0<m<1$, such that

$$
\phi(c, d, u, v) \leq 4 m \phi\left(\frac{c}{2}, \frac{d}{2}, \frac{u}{2}, \frac{v}{2}\right)
$$

for all $c, d, u, v \in \mathcal{A}$, then there exists a unique quadratic double centralizer $(L, R)$ on $\mathcal{A}$ satisfying

$$
\begin{aligned}
& \left\|f_{0}(a)-L(a)\right\| \leq \frac{1}{4(1-m)} \phi(a, a, 0,0), \\
& \left\|f_{1}(a)-R(a)\right\| \leq \frac{1}{4(1-m)} \phi(a, a, 0,0)
\end{aligned}
$$

for all $a \in \mathcal{A}$.

Proof. From (2.3), it follows that

$$
\lim _{i} 4^{-i} \phi\left(2^{i} c, 2^{i} d, 2^{i} u, 2^{i} v\right)=0
$$

for all $c, d, u, v \in \mathcal{A}$. Putting $j=0, \lambda=1, a=b$ and replacing $a$ by $2 a$ in (2.1), we get

$$
\left\|f_{0}(2 a)-4 f_{0}(a)\right\| \leq \phi(a, a, 0,0)
$$

for all $a \in \mathcal{A}$. By the above inequality, we have

$$
\left\|\frac{1}{4} f_{0}(2 a)-f_{0}(a)\right\| \leq \frac{1}{4} \phi(a, a, 0,0)
$$

for all $a \in \mathcal{A}$. Consider the set $X:=\{g \mid g: \mathcal{A} \rightarrow \mathcal{A}\}$ and introduce the generalized metric on $X$ :

$$
d(h, g):=\inf \left\{C \in \mathbb{R}^{+}:\|g(a)-h(a)\| \leq C \phi(a, a, 0,0) \text { for all } a \in \mathcal{A}\right\} .
$$

It is easy to show that $(X, d)$ is complete. Now, we define the mapping $Q: X \rightarrow X$ by

$$
Q(h)(a)=\frac{1}{4} h(2 a)
$$


for all $a \in \mathcal{A}$. Given $g, h \in X$, let $C \in \mathbb{R}^{+}$be an arbitrary constant with $d(g, h) \leq C$, that is,

$$
\|g(a)-h(a)\| \leq C \phi(a, a, 0,0)
$$

for all $a \in \mathcal{A}$. Substituting $a$ by $2 a$ in the inequality (2.9) and using (2.3) and (2.8), we have

$$
\begin{aligned}
\|(Q g)(a)-(Q h)(a)\| & =\frac{1}{4}\|g(2 a)-h(2 a)\| \\
& \leq \frac{1}{4} C \phi(2 a, 2 a, 0,0) \\
& \leq C m \phi(a, a, 0,0)
\end{aligned}
$$

for all $a \in \mathcal{A}$. Hence, $d(\mathrm{Qg}, \mathrm{Q} h) \leq \mathrm{Cm}$. Therefore, we conclude that $d(\mathrm{Qg}, \mathrm{Qh}) \leq m d$ $(g, h)$ for all $g, h \in X$. It follows from (2.7) that

$$
d\left(Q f_{0}, f_{0}\right) \leq \frac{1}{4}
$$

By Theorem 2.1, $Q$ has a unique fixed point $L: \mathcal{A} \rightarrow \mathcal{A}$ in the set $=\left\{h \in X, d\left(f_{0}, h\right)\right.$ $<\infty$. On the other hand,

$$
\lim _{n \rightarrow \infty} \frac{f_{0}\left(2^{n} a\right)}{4^{n}}=L(a)
$$

for all $a \in \mathcal{A}$. By Theorem 2.1 and (2.10), we obtain

$$
d\left(f_{0}, L\right) \leq \frac{1}{1-m} d\left(Q f_{0}, L\right) \leq \frac{1}{4(1-m)},
$$

i.e., the inequality (2.4) is true for all $a \in \mathcal{A}$. Now, substitute $2^{n} a$ and $2^{n} b$ by $a$ and $b$, respectively, and put $j=0$ in (2.1). Dividing both sides of the resulting inequality by $2^{n}$, and letting $n$ go to infinity, it follows from (2.6) and (2.11) that

$$
L(\lambda a+\lambda b)+L(\lambda a-\lambda b)=2 \lambda^{2} L(a)+2 \lambda^{2} L(b)
$$

for all $a, b \in \mathcal{A}$ and $\lambda \in \mathbb{T}$. Putting $\lambda=1$ in (2.12), we have

$$
L(a+b)+L(a-b)=2 L(a)+2 L(b)
$$

for all $a, b \in \mathcal{A}$. Hence, $L$ is a quadratic mapping.

Letting $b=0$ in (2.13), we get $L(\lambda a)=\lambda^{2} L(a)$ for all $a, b \in \mathcal{A}$ and $\lambda \in \mathbb{T}$. By (2.13), $L$ $(r a)=r^{2} L(a)$ for any rational number $r$. It follows from the continuity $f_{0}$ and $\varphi$ for each $\lambda \in \mathbb{R}, L(\lambda a)=\lambda^{2} L(a)$. Hence,

$$
L(\lambda a)=L\left(\frac{\lambda}{|\lambda|}|\lambda| a\right)=\frac{\lambda^{2}}{|\lambda|^{2}} L(|\lambda| a)=\frac{\lambda^{2}}{|\lambda|^{2}}|\lambda|^{2} L(a)
$$

for all $a \in \mathcal{A}$ and $\lambda \in \mathbb{C}(\lambda \neq 0)$. Therefore, $L$ is quadratic homogeneous. Putting $j=$ $0, u=v=0$ in (2.2) and replacing $2^{n} c$ by $c$, we obtain

$$
\left\|\frac{f_{0}\left(2^{n} c d\right)}{4^{n}}-\frac{f_{0}\left(2^{n} c\right)}{4^{n}} d\right\| \leq \frac{1}{2} 4^{-n} \phi\left(2^{n} c, d, 0,0\right) .
$$


By (2.6), the right-hand side of the above inequality tend to zero as $n \rightarrow \infty$. It follows from (2.11) that $L(c d)=L(c) d^{2}$ for all $c, d \in \mathcal{A}$. Thus, $L$ is a quadratic left centralizer.

Also, one can show that there exists a unique mapping $R: \mathcal{A} \rightarrow \mathcal{A}$ which satisfies

$$
\lim _{n \rightarrow \infty} \frac{f_{1}\left(2^{n} a\right)}{4^{n}}=R(a)
$$

for all $a \in \mathcal{A}$. The same manner could be used to show that $R$ is quadratic right centralizer. If we substitute $u$ and $v$ by $2^{n} u$ and $2^{n} v$ in (2.2), respectively, and put $c=d=$ 0 , and divide the both sides of the obtained inequality by $16^{n}$, then we get

$$
\left\|u^{2} \frac{f_{0}\left(2^{n} v\right)}{4^{n}}-\frac{f_{1}\left(2^{n} u\right)}{4^{n}} v^{2}\right\| \leq 16^{-n} \phi\left(0,0,2^{n} u, 2^{n} v\right) \leq 4^{-n} \phi\left(0,0,2^{n} u, 2^{n} v\right)
$$

Passing to the limit as $n \rightarrow \infty$, and again from (2.5) we conclude that $u^{2} L(v)=R(u)$ $v^{2}$ for all $u, v \in \mathcal{A}$. Therefore, $(L, R)$ is a quadratic double centralizer on $\mathcal{A}$. This completes the proof of the theorem.

\section{Stability of quadratic multipliers}

Assume that $\mathcal{A}$ is a complex Banach algebra. Recall that a mapping $T: \mathcal{A} \rightarrow \mathcal{A}$ is a quadratic multiplier if $T$ is a quadratic homogeneous mapping, and $a^{2} T(b)=T(a) b^{2}$ for all $a, b \in \mathcal{A}$ (see [21]). We investigate the stability of quadratic multipliers.

Theorem 3.1. Let $f: \mathcal{A} \rightarrow \mathcal{A}$ be a continuous mapping with $f(0)=0$ and let $\phi: \mathcal{A}^{4} \rightarrow[0, \infty)$ be a continuous in the first and second variables such that

$$
\begin{gathered}
\left\|f(a+\lambda b)+f(\lambda a-\lambda b)-2 \lambda^{2}[f(a)+f(b)]+c^{2} f(d)-f(c) d^{2}\right\| \\
\leq \phi(a, b, c, d)
\end{gathered}
$$

for all $\lambda \in \mathbb{T}$ and all $a, b, c, d \in \mathcal{A}$.If there exists a constant $m, 0<m<1$, such that

$$
\phi(2 a, 2 b, 2 c, 2 d) \leq 4 m \phi(a, b, c, d)
$$

for all $a, b, c, d \in \mathcal{A}$. Then, there exists a unique quadratic multiplier $T$ on $\|f(a)-T(a)\| \leq \frac{1}{4(1-m)} \phi(a, a, 0,0)$ satisfying

$$
\|f(a)-T(a)\| \leq \frac{1}{4(1-m)} \phi(a, a, 0,0)
$$

for all $a \in \mathcal{A}$.

Proof. It follows from $\varphi(2 a, 2 b, 2 c, 2 d) \leq 4 m \varphi(a, b, c, d)$ that

$$
\lim _{n \rightarrow \infty} \frac{\phi\left(2^{n} a, 2^{n} b, 2^{n} c, 2^{n} d\right)}{4^{n}}=0
$$

for all $a, b, c, d \in \mathcal{A}$. Putting $\lambda=1, a=b, c=d, d=0$ in (3.1), we obtain

$$
\|f(2 a)-4 f(a)\| \leq \phi(a, a, 0,0)
$$

for all $a \in \mathcal{A}$. Hence,

$$
\left\|f(a)-\frac{1}{4} f(2 a)\right\| \leq \frac{1}{4} \phi(a, a, 0,0)
$$


for all $a \in \mathcal{A}$. Consider the set $X:=\{h \mid h: \mathcal{A} \rightarrow \mathcal{A}\}$ and introduce the generalized metric on $X$ :

$$
d(g, h):=\inf \left\{C \in \mathbb{R}^{+}:\|g(a)-h(a)\| \leq C \phi(a, a, 0,0) \text { for all } a \in \mathcal{A}\right\} .
$$

It is easy to show that $(X, d)$ is complete. Now, we define a mapping $\Phi: X \rightarrow X$ by

$$
\Phi(h)(a)=\frac{1}{4} h(2 a)
$$

for all $a \in \mathcal{A}$. By the same reasoning as in the proof of Theorem 2.2, $\Phi$ is strictly contractive on $X$. It follows from (3.5) that $d(\Phi f, f) \leq \frac{1}{4}$. By Theorem 2.1, $\Phi$ has a unique fixed point in the set $X_{1}=\{h \in X: d(f, h)<\infty\}$. Let $T$ be the fixed point of $\Phi$. Then, $T$ is the unique mapping with $T(2 a)=4 T(a)$, for all $a \in \mathcal{A}$ such that there exists $C \in(0, \infty)$ such that

$$
\|T(x)-f(x)\| \leq C \phi(a, a, 0,0)
$$

for all $a \in \mathcal{A}$. On the other hand, we have $\lim _{n \rightarrow \infty} d\left(\Phi^{n}(f), T\right)=0$.

Thus,

$$
\lim _{n \rightarrow \infty} \frac{1}{4^{n}} f\left(2^{n} x\right)=T(x)
$$

for all $a \in \mathcal{A}$. Hence,

$$
d(f, T) \leq \frac{1}{1-m} d(T, \Phi(f)) \leq \frac{1}{4(1-m)} .
$$

This implies the inequality (3.3). It follows from (3.1), (3.4) and (3.6) that

$$
\begin{gathered}
\left\|T(\lambda a+\lambda b)+T(\lambda a-\lambda b)-2 \lambda^{2} T(a)-2 \lambda^{2} T(b)\right\| \\
=\lim _{n \rightarrow \infty} \frac{1}{4^{n}}\left\|T\left(2^{n}(\lambda a+\lambda b)\right)+T\left(2^{n}(\lambda a-\lambda b)\right)-2 \lambda^{2} T\left(2^{n} a\right)-2 \lambda^{2} T\left(2^{n} b\right)\right\| \\
\leq \lim _{n \rightarrow \infty} \frac{1}{4^{n}} \phi\left(2^{n} a, 2^{n} b, 0,0\right)=0
\end{gathered}
$$

for all $a, b \in \mathcal{A}$. Hence,

$$
T(\lambda a+\lambda b)+T(\lambda a-\lambda b)=2 \lambda^{2} T(a)+2 \lambda^{2} T(b)
$$

for all $a, b \in \mathcal{A}$ and $\lambda \in \mathbb{T}$. Letting $b=0$ in (3.8), we have $T(\lambda a)=\lambda^{2} T(a)$, for all $a, b \in \mathcal{A}$ and $\lambda \in \mathbb{T}$. Now, it follows from the proof of Theorem 2.1 and the continuity $f$ and $\varphi$ that $T$ is $\mathbb{C}$-linear. If we substitute $c$ and $d$ by $2^{n} c$ and $2^{n} d$ in (3.1), respectively, and put $a=b=0$ and we divide the both sides of the obtained inequality by $16^{n}$, we get

$$
\left\|c^{2} \frac{f\left(2^{n} d\right)}{4^{n}}-\frac{f\left(2^{n} c\right)}{4^{n}} d^{2}\right\| \leq \frac{\phi\left(0,0,2^{n} c, 2^{n} d\right)}{16^{n}} \leq \frac{\phi\left(0,0,2^{n} c, 2^{n} d\right)}{4^{n}} .
$$

Passing to the limit as $n \rightarrow \infty$, and from (3.4) we conclude that $c^{2} T(d)=T(c) d^{2}$ for all $c, d \in \mathcal{A}$. 


\section{Author details}

'Department of Mathematics, Research Institute for Natural Sciences, Hanyang University, Seoul 133-791, Korea

${ }^{2}$ Department of Mathematics, Daejin University, Kyeonggi 487-711, Korea ${ }^{3}$ Department of Mathematics, University of

Seoul, Seoul 130-743, Korea ${ }^{4}$ Department of Mathematics, Semnan University, P. O. Box 35195-363, Semnan, Iran

\section{Authors' contributions}

All authors conceived of the study, participated in its design and coordination, drafted the manuscript, participated in the sequence alignment, and read and approved the final manuscript.

\section{Competing interests}

The authors declare that they have no competing interests.

Received: 13 July 2011 Accepted: 1 November 2011 Published: 1 November 2011

\section{References}

1. Ulam, SM: Problems in Modern Mathematics, Chapter VI, Science edn. Wiley, New York (1940)

2. Hyers, DH: On the stability of the linear functional equation. Proc Nat Acad Sci USA. 27, 222-224 (1941). doi:10.1073/ pnas.27.4.222

3. Rassias, ThM: On the stability of the linear mapping in Banach spaces. Proc Am Math Soc. 72, 297-300 (1978). doi:10.1090/50002-9939-1978-0507327-1

4. Găvruta, P: A generalization of the Hyers-Ulam-Rassias stability of approximately additive mappings. J Math Anal Appl. 184, 431-436 (1994). doi:10.1006/jmaa.1994.1211

5. Skof, F: Propriet locali e approssimazione di operatori. Rend Sem Mat Fis Milano. 53, 113-129 (1983). doi:10.1007/ BF02924890

6. Cholewa, PW: Remarks on the stability of functional equations. Aequationes Math. 27, $76-86$ (1984). doi:10.1007/ BF02192660

7. Czerwik, S: On the stability of the quadratic mapping in normed spaces. Abh Math Sem Univ Hamburg. 62, 59-64 (1992). doi:10.1007/BF02941618

8. Eshaghi Gordji, M, Bodaghi, A: On the Hyers-Ulam-Rassias stability problem for quadratic functional equations. East J Approx. 16, 123-130 (2010)

9. Eshaghi Gordji, M, Moslehian, MS: A trick for investigation of approximate derivations. Math Commun. 15, 99-105 (2010)

10. Eshaghi Gordji, M, Rassias, JM, Ghobadipour, N: Generalized Hyers-Ulam stability of generalized $(n, k)$-derivations. Abstr Appl Anal 8 (2009). Article ID 437931

11. Eshaghi Gordji, M, Khodaei, H: Solution and stability of generalized mixed type cubic, quadratic and additive functional equation in quasi-Banach spaces. Nonlinear Anal TMA. 71, 5629-5643 (2009). doi:10.1016/j.na.2009.04.052

12. Kannappan, PI: Quadratic functional equation and inner product spaces. Results Math. 27, 368-372 (1995)

13. Moslehian, MS, Najati, A: An application of a fixed point theorem to a functional inequality. Fixed Point Theory. 10, 141-149 (2009)

14. Najati, A, Park, C: Fixed points and stability of a generalized quadratic functional equation. J Inequal Appl 19 (2009). Article ID 193035

15. Najati, A, Park, C: The pexiderized Apollonius-ensen type additive mapping and isomorphisms between $C^{*}$-algebras. J Diff Equa Appl. 14, 459-479 (2008). doi:10.1080/10236190701466546

16. Najati, A: Hyers-Ulam stability of an n-Apollonius type quadratic mapping. Bull. Belg Math Soc Simon Stevin. 14 755-774 (2007)

17. Najati, A: Homomorphisms in quasi-Banach algebras associated with a pexiderized Cauchy-Jensen functional equation. Acta Math Sin Engl Ser. 25(9), 1529-1542 (2009). doi:10.1007/s10114-009-7648-z

18. Lee, J, An, J, Park, C: On the stability of quadratic functional equations. Abstr Appl Anal 8 (2008). Article ID 628178

19. Baker, J: The stability of the cosine equation. Proc Am Math Soc. 80, 242-246 (1979)

20. Eshaghi Gordji, M, Bodaghi, A: On the stability of quadratic double centralizers on Banach algebras. J Comput Anal Appl. 13, 724-729 (2011)

21. Eshaghi Gordji, M, Ramezani, M, Ebadian, A, Park, C: Quadratic double centralizers and quadratic multipliers. Ann Univ Ferrara. 57, 27-38 (2011). doi:10.1007/s11565-011-0115-7

22. Diaz, J, Margolis, B: A fixed point theorem of the alternative for contractions on a generalized complete metric space. Bull Am Math Soc. 74, 305-309 (1968). doi:10.1090/S0002-9904-1968-11933-0

doi:10.1186/1029-242X-2011-104

Cite this article as: Park et al.: Comment on "on the stability of quadratic double centralizers and quadratic multipliers: a fixed point approach" [Bodaghi et al., j. inequal. appl. 2011, article id 957541 (2011)]. Journal of Inequalities and Applications 2011 2011:104. 